\title{
Philosophiques
}

\section{Le continuum corps-esprit dans l'économie de notre être selon Bonnet}

\section{John O’Neal}

Volume 19, numéro 1, printemps 1992

URI : https://id.erudit.org/iderudit/027174ar

DOI : https://doi.org/10.7202/027174ar

Aller au sommaire du numéro

Éditeur(s)

Société de philosophie du Québec

ISSN

0316-2923 (imprimé)

1492-1391 (numérique)

Découvrir la revue

Citer cet article

O’Neal, J. (1992). Le continuum corps-esprit dans l'économie de notre être selon Bonnet. Philosophiques, 19(1), 87-111. https://doi.org/10.7202/027174ar d'utilisation que vous pouvez consulter en ligne.

https://apropos.erudit.org/fr/usagers/politique-dutilisation/ 
PHILOSOPHIQUES, VOL. XIX, NUMÉRO 1. PRINTEMPS 1992

\title{
INTERVENTION
}

\section{LE CONTINUUM CORPS-ESPRIT DANS L'ÉCONOMIE DE NOTRE ÊTRE SELON BONNET}

\author{
par John O'Neal
}

Peu après qu'il eût entamé la rédaction de son Essai analytique sur les facultés de l'âme (publié finalement en 1760) se servant d'une statue imaginaire pour expliquer la genèse du savoir humain, Charles Bonnet découvrit que Condillac avait déjà exploité cette construction de l'esprit dans son Traité des sensations ${ }^{1}$. Toutefois, plutôt que d'abandonner son projet, Bonnet décida de le mener à bien, après avoir étudié avec soin le traité de Condillac, estimant qu'il faisait un usage qualitativement différent de la statue et apportait par conséquent sa propre contribution significative à l'épistémologie du dix-huitième siècle. Son originalité résidait, croyait-il, en une approche analytique qui, selon ses observations, faisait défaut dans l'œuvre de Condillac [11,

NOTE DE LA RÉDACTION: Charles Bonnet, philosophe et naturaliste genevois $\mathrm{du}$ siècle des Lumières, est essentiel pour comprendre le passage d'une anthropologie captive de l'âme à une science matérialiste du corps qui ne parvient pas tout à fait, aujourd'hui encore, à se débarasser des éléments idéalistes qui le parasitent.

John O'Neal, éminent spécialiste du sensualisme et de la pensée du XVIIIe siècle, expose ici en historien des idées, les grands thèmes du projet physiologique et philosophique de Bonnet marquant la continuité entre sensation et âme, entre corps et esprit: la "Chaîne des Êtres ", les liens de l'homme avec l'animal, en particulier avec le singe, la nature mixte de l'homme, la "succession" dans nos procédés cognitifs, le "principe volontaíre" ramené au déterminisme dynamique des sens.

Les lecteurs de Philosophiques seront sans doute curieux de connaître, selon la perspective que développe un Bonnet, "charnière " entre un évolutionisme statique, déterministe et matérialiste et un évolutionisme empirique et dynamique, une des grandes sources dix-huitièmistes avec Helvétius, Condillac et Diderot, de ce problème des relations corps-esprit qui se retrouve au cœur de presque tous nos débats théoriques contemporains. 
un ouvrage précédent intitulé Essai de psychologie (1754)2, Bonnet ne développa pas complètement ses propres idées sur la question avant l'Essai analytique.

En composant cette dernière œuvre, Bonnet se donne pour sujet de recherche ce qu'il dénomme "l'CEconomie de nôtre Être » $[v, x I I I, x x I]$. Par ces termes évocateurs, Bonnet entend essentiellement l'étude des modifications positives et négatives dans le corps, le cerveau et l'âme. Le concept, bien que mentionné maintes fois dans la préface, n'est ni pleinement appréhensible, ni répété en ces termes avant les dernières pages de l'essai [355-56, 370, 473] et dans sa conclusion [538]. Le moment où Bonnet $s^{\prime}$ étend sur l'économie de notre être coïncide, on $s^{\prime} y$ attendra, avec la concentration de l'intérêt sur un moment critique antérieur dans son analyse des opérations de l'âme, un moment où les sensations s'accumulent ou se perdent pour la première fois. Rétrospectivement, $c^{\prime}$ est un moment crucial à la fois du point de vue textuel et intertextuel, puisque non seulement il confère unité et cohérence à l'œuvre de Bonnet, mais il permet également de la différencier nettement de celle de Condillac. Alors que Condillac fait tour à tour exercer à la statue un sens puis un autre et lui fait éprouver de nombreuses sensations différentes, Bonnet se concentre principalement sur les deux sensations originelles qui dériveront chez la statue du sens olfactif. Cette période cruciale expliquera le fonctionnement des facultés, l'évolution des idées et révélera finalement la profondeur de la méthode analytique de Bonnet ainsi que de son sensualisme qui fondamentalement sont tous deux en corrélation étroite dans un continuum du corps et de l'esprit. Mais avant de s'y consacrer, Bonnet avance un certain nombre d'hypothèses générales qui lui permettent d'organiser un tel argument. Parmi elles, la nécessité de l'âme, la nature mixte de l'homme, la loi de l'union, la prédominance du physique sur la métaphysique et l'importance de la succession dans la cognition humaine.

Lorsqu'il conclut la préface de son Essai analytique, Bonnet justifie la nécessité de l'âme: "Ce n'est point parce que je crois l'Âme un Être plus excellent que la Matière, que j'attribuë une Âme à l'Homme: c'est uniquement, parce que je ne puis attribuer à la Matiére tous les Phénomenes de l'Homme" [xxiv]. C'est la nature immatérielle de l'âme qui amène Bonnet à lui consacrer une place dans son ontologie. Son existence est en outre "fondé[e] sur l'opposition qui est entre la simplicité du sentiment \& la composition de la Matiere » [3]. L'âme, mieux que tout autre chose, explique cette simplicité du sentiment: 
"C'est pour satisfaire à ce Sentiment du Moi, toûjours un, toûjours simple, toûjours indivisible, que nous recourrons à l'existence de cette Substance immatérielle que nous nommons l'Âme" [297]. Elle procure également au corps une force motrice interne et aide Bonnet à esquiver commodément toute association avec le matérialisme qui heurterait ses convictions religieuses [EA 86, 296; EP vil f].

Si l'apport de l'âme dans l'équation épistémologique résout quelques problèmes, il en soulève de nouveaux. Un des non moins épineux sera celui de la nature de cette âme. Dans l'Essai de psychologie, Bonnet tente d'esquiver le sujet en se déclarant d'une totale ignorance en ce domaine [EP 2]. C'est une attitude qu'il avait également adoptée face à d'autres sujets, notamment dans la façon dont le mouvement d'une fibre fait naître une idée dans l'âme, ou la nature du mouvement et des autres forces dans la matière [EP 105, 118]. Bonnet ne tente pas plus de situer ou d'identifier le siège de l'âme mais recourt à des métaphores mécaniques pour le décrire, telles le clavecin, l'orgue ou l'horloge [EP 13].

Une forme de définition relative de l'âme finit pourtant par transparaître dans les écrits de Bonnet, dérivant de ce qu'il appelle la nature mixte de l'homme et la loi de l'union. Dans l'Essai de psychologie, il écrit, à propos de la nature mixte des êtres humains: « l'Homme est de sa Nature un être mixte, un être composé nécessairement de deux substances, l'une spirituelle, l'autre corporelle " [EP 3]. Ce n'est pas avant un autre ouvrage de la même période, Principes philosophiques sur la cause première et sur son effet, que Bonnet approfondit ses observations sur notre nature mixte et tente d'élucider sa complexité ${ }^{3}$. Bien qu'il entoure l'union des deux substances d'une «épaisse nuit » et d'un profond mystère [EP 123, 168] - les nœuds qui les unissent ne sont connus que de leur créateur - une loi gouvernant l'union du corps et de l'âme peut être énoncée: "La Loi Fondamentale de cette Union est, qu'à l'occasion des Mouvemens qui s'excitent dans le Corps, l'Âme est modifiée; \& qu'à l'occasion des modifications de l'Âme, le Corps est $m \hat{u}$ " [PP 298-99; EA 29]. À chaque mouvement corporel correspond une modification de l'âme, et à chaque modification de l'âme correspond un mouvement corporel. Les substances matérielles et immatérielles sont en état de mutuelle interdépendance, l'une ne pouvant se définir sans l'autre. 
Les affirmations de Bonnet concernant notre nature mixte et la loi de l'union sont à l'origine de son sensualisme et de sa préoccupation pour le monde physique. Si l'accent qu'il porte à la physiologie humaine le rallie toujours davantage à un point de vue sensualiste, il le différencie néanmoins considérablement de Condillac et d'Helvétius qui s'abstiennent généralement de toute référence aux parties anatomiques du système nerveux. En un mot, il est davantage neuro-physicien que métaphysicien. Dans un même passage de sa préface à l'Essai analytique, il s'attribue le statut d'investigateur en matière d'économie de notre être, rappelle les deux précédentes assertions relatives au corps et à l'âme, et fait présager une approche résolument sensualiste du sujet.

J'ai mis dans mon Livre beaucoup de Physique, \& assez peu de Métaphysique: mais, en vérité, que pouvois-je dire de l'Âme considérée en elle-même? Nous la connoissons si peu. L'Homme est un Être mixte; il n'a des Idées que par l'intervention des sens, \& ses Notions les plus abstraites dérivent encore des Sens. C'est sur son Corps, \& par son Corps que l'Âme agit. Il faut donc toûjours en revenir au Physique, comme à la prémiere origine de tout ce que l'Âme éprouve [XIII].

Le physique influe sur toutes les opérations de l'âme [12]. Bien qu'il nous avertisse de prendre au sens figuré certains des termes physiques qu'il utilise, du fait de la pauvreté du vocabulaire propre à l'esprit quelle que soit la langue, Bonnet attribue une toute puissance à cet aspect matériel de notre être [85-86]. Il servira en réalité à expliquer l'autre aspect.

Pour appréhender la méthode de Bonnet, il faut procéder analytiquement, comme lui, en simplifiant et décomposant par degrés et successivement, du connu vers l'inconnu, du simple vers le composé $[E A 1-2,7,65]^{4}$. En ce qui concerne le monde physique, une telle approche associe constamment le corps - ses sensations, ses organes et ses fibres - à des facultés d'ordre supérieur. Il en résulte une hiérarchie épistémologique. Bonnet a lui-même recours à une terminologie de domination dans un système vraisemblablement régi par la sagesse du divin auteur de la nature qui a "subordonné l'Activité de l'Âme à sa Sensibilité; sa sensibilité au Jeu des Fibres; le Jeu des Fibres à l'Action des Objets » [86-87, 92]. Il est implicite que les idées "dans leur prémiere origine" ne sont que «les mouvemens imprimés par les Objets aux Fibres des Sens" [40]. La liberté sera subordonnée à la volonté, qui à son tour sera subordonnée à la faculté de sentir ou de 
savoir et ainsi de suite [116]. L'ordre du mouvement est donc le suivant: objets, organes (fibres), sensations ou perceptions, volonté, liberté. Si Bonnet choisit d'y revenir aussi fréquemment [86-87, 115-116, 258, 287], c'est que cet ordre ou hiérarchie constitue la "Base de toute la Science de nôtre Être" [288].

À plusieurs reprises dans l'Essai analytique, Bonnet réitère l'importance de la succession dans la cognition humaine. L'idée est en réalité étroitement liée à celle de continuité et de gradation développée dans ses autres ouvrages ${ }^{5}$. Après que la statue découvre pour la première fois la sensation d'une odeur, celle de la rose, cette sensation ne se dissipe que "par degrés " ou "par gradations" [32]. La temporalité même est définie en termes de succession d'idées [72]. L'imagination reproduira les idées dans un ordre grandement influencé par celui dans lequel elles se sont initialement succédées [157]. La succession des êtres permet à l'esprit d'appréhender la notion de priorité et de postériorité [171]. En un mot, notre existence même est successive.

$\mathrm{Au}$ fil de sa démarche analytique, Bonnet n'établit rien de moins qu'un enchaînement cognitif d'événements dont l'essentielle spécificité se trouve être leur nature successive. Selon Bonnet, "le véritable ornement d'une Analyse consiste dans la vérité, la netteté \& l'enchaînement des Idées " [XII]. Les « idées » mises en rapport dans l'analyse de Bonnet représentent les étapes d'un processus cognitif qui conduit ironiquement aux idées dont il essaie précisément de retrouver l'origine dans l'âme. Les procédés de la nature qui n'avance pas "par sauts " mais "par degrés» deviennent également ses procédés d'analyse [30].

L'exposé précédent prépare le moment crucial où deux sensations se succèdent chez la statue. Au chapitre XXI qui devait à l'origine conclure $l^{\prime}$ Essai analytique ${ }^{6}$ - Bonnet se voit entraîné assez loin par sa méthode analytique et se résigne volontiers à reconnaître "que deux Sensations suffisoient à mettre en jeu toutes les Facultés de l'Âme de ma Statuë " [355]. La surprise de Bonnet comme auteur lorsqu'il évalue rétrospectivement la distance parcourue est encore plus vive chez le lecteur. C'est le lecteur qui doit faire marche arrière et réorganiser les diverses articulations de l'argument en tenant compte de l'éclaircissement apporté dans le contexte d'une réévocation spécifique de l'économie de notre être. En fin de compte, l'économie de notre être, que Bonnet déclare prendre 
comme sujet de ses recherches, est en étroite corrélation avec la succession des sensations qui dans un esprit sensualiste la définit en grande partie. Bonnet élabore cette économie principalement au niveau des deux premières sensations éprouvées par la statue. À partir de celles-ci, il expliquera non seulement le fonctionnement d'un certain nombre de facultés, attributs et sentiments humains tels la mémoire, la personnalité, l'imagination, le plaisir et la douleur, l'attention, la volonté, la liberté et le désir, mais également l'évolution des idées ${ }^{7}$. Un coup d'œil à l'exposé de Bonnet sur les états initiaux chez la statue s'avère ici utile.

Bonnet examine tout d'abord la statue avant qu'elle n'ait commencé à sentir et parvient à cette conclusion sensualiste qu'en l'absence de sensations, elle n'a point d'idées [13]. Lorsque la statue commence à sentir, Bonnet, suivant l'exemple de Condillac, lui fait exercer en premier le sens olfactif du fait que c'est le sens le plus simple et le mieux approprié pour sa méthode analytique [25]. Il examine ensuite sa statue après la première sensation, qui est bien sûr l'odeur d'une rose, reconnaissant que le rappel des idées sous-entend des relations avec d'autres idées et que, par conséquent, un cerveau doté d'une seule idée ne peut la rappeler [35].

La mémoire pourtant, comme toutes les autres facultés dans l'ouvrage de Bonnet, est liée au corps et peut donc recevoir une explication physiologique [39]. Avant même d'éprouver la seconde sensation, la statue a déjà, dans une certaine mesure, fait l'expérience de l'économie de son être, qui est exprimée en termes mathématiques et tangibles de perte et de gain: "L'état primitif des Fibres sur lesquelles ces Corpuscules ont agi pendant un certain tems a été modifié, \& cette modification est l'expression physique de la différence qui est entre l'état actuel de nôtre Statuë \& celui qui avait précedé la Sensation » [43]. Ce n'est toutefois qu'avec la succession des sensations les unes après les autres que l'économie de l'être de la statue se dégage ostensiblement et que son existence est réaffirmée, car sentir est indispensable à un être sentant. En l'absence de sensibilité ou de faculté de sentir, il ne peut en aucun cas exister [44]. Bonnet va jusqu'à poser cette question rhétorique: «la vie de l'Âme est-elle autre chose que la succession de ces idées rappellées les unes par les autres? " [45].

En se faisant succéder les sensations chez la statue, Bonnet vise à répondre à une importante question relative à la mémoire 
et la réciprocité des actions entre les fibres de différentes natures; pour être précis: "l'odeur de l'Oeillet rappellera-telle à nôtre Statuë celle de la Rose? » [54]. Bien que l'on soit tenté de donner une réponse négative à cette question, l'expérience nous foumit des faits qui corroborent la réciprocité d'action [55]. La "condition essentielle» au rappel des sensations est "que les Fibres sur lesquelles d'autres Fibres agissent ayent été muës auparavant par les Objets » [56]. La réminiscence consiste en l'impression de sentir à nouveau une modification que l'âme a déjà sentie [59, 346]. Son effet est $d^{\prime}$ " instruire l'Âme de l'identité, ou de la diversité de ses modifications" [62]. Bonnet remarque que cela constitue « un des points les plus importants de l'œconomie de nôtre Être, mais qu'il n'est pas tems encore de discuter " [ibid.]. Il n'examinera l'économie de notre être que plus tard, mais l'on peut affirmer rétrospectivement que la réminiscence indique à l'âme - si la modification dont il est l'objet génère un sentiment d'identité ou de différence - le résultat d'un gain ou d'une perte. La réminiscence permet en outre à la statue de sentir "la nouveauté de sa situation" lorsqu'une seconde odeur lui est présentée [ibid.]. La première sensation d'odeur de rose est en quelque sorte un point de repère auquel seront comparées les sensations subséquentes.

Dans sa démarche qui relève à la fois de la physiologie et $\mathrm{du}$ sensualisme, Bonnet explique la réminiscence en termes de fibres. C'est le corps et non l'âme qui rend la réminiscence possible: "La Mémoire, par laquelle nous retenons les Idées des Choses, a été attachée au Corps " [39]. L'âme peut sentir mais "c'est toûjours le Corps qui fait sentir» [64]. Les liens qui unissent les idées aux sensations par l'entremise de la réminiscence - et l'esprit au corps - sont resserrés: «Les Idées n'étant dans leur prémiere origine que les mouvemens imprimés par les Objets aux Fibres des Sens, il s'ensuit que la conservation des Idées par la Mémoire dépend en dernier ressort de la disposition qu'ont les Fibres des Sens à se prêter à ces mouvemens \& à les répeter" [40]. Bonnet progresse d'allusions générales et indirectes à propos du corps (sensations), vers des allusions anatomiques et physiques de plus en plus spécitiques (fibres, molécules, sucs nutritifs et atomes [6469]).

De même qu'il existe une économie de notre être, ainsi existe-t-il une économie des fibres qui conservent toute configuration que leur ont imprimé les objets [64f]. Dans l'une des rares 
références positives à l'Essai de Psychologie - il s'agit ici d'une citation - Bonnet déclare: "La répétition fréquente du même mouvement dans la même Fibre change jusqu'à un certain point l'état primitif de cette Fibre" [EA 69-70; EP 206]. Les fibres gardent donc une empreinte physique et peuvent retransmettre à l'âme le souvenir d'une impression qu'un objet a laissée sur les sens [EA 74, 383-84, 442]. Alors que le souvenir d'une impression et la sensation originale ont la même source (la fibre), il est plus faible que la sensation [366]. Mais il n'y aurait point de réminiscence chez un être qui éprouverait toute sa vie la même sensation au même degré puisque la réminiscence "suppose dans l'Âme un changement d'état, une succession de modifications" [71-72].

Une telle définition de la réminiscence peut porter à croire que les fibres, une fois organisées selon une certaine configuration, se maintiennent ainsi à jamais. De nombreuses forces telles "les mouvements intestins" et les "petites impulsions étrangeres aux Objets \& à l'Âme" ainsi que les effets des systèmes circulatoires et nutritifs " concourent à chaque instant à changer l'état actuel des Fibres des Sens" [75]. De même que les fibres sont formées pour conserver la réminiscence, elles " $\left[s^{\prime}\right]$ effaceront » aussi, soit par dégénérescence ou usage, ainsi qu'il en va dans tout corps animal, soit sous l'effet $d^{\prime}$ impulsions étrangères perturbatrices [75-76]. Une économie de croissance peut faire place à une économie de perte. Quoi qu'il en soit, chaque économie joue un rôle important dans la constitution de notre être. Le total de ces diverses modifications par addition ou soustraction influence largement, voire même dicte en réalité la structure de notre personnalité.

La personnalité prend son fondement dans la mémoire qui, ainsi que le montre Bonnet, prend elle-même son fondement dans la succession des sensations au coeur de l'économie de notre être. Lorsqu'il s'apprête à conclure le chapitre IX [5983] qui contient son "Essai d'une Théorie de la Réminiscence" Bonnet écrit: «Lors donc que l'Âme éprouve l'impression d'un Objet, \& qu'elle se rappelle en même tems une, ou plusieurs autres impressions, elle s'identifie avec toutes; \& cette identification est le fondement de la Personalité " [80]. Bonnet distingue toutefois deux formes différentes de personnalité: une première qui pourrait s'appliquer aux animaux puisqu'elle "résulte simplement de la liaison que la Réminiscence met entre les Sensations antécédentes \& les Sensations subséquentes, en vertu de laquelle l'Âme a le sentiment des changements d'état par lesquels elle passe"; 
une seconde qui est réfléchie et " consiste dans ce retour de l'Âme sur elle-même, par lequel séparant en quelque sorte de soi ses propres Sensations, elle réfléchit que c'est elle qui les éprouve, ou qui les a éprouvé " [80-81]. Il existe bien entendu une différence entre réflexion et sensation ${ }^{8}$. La réflexion suppose l'usage de signes, dans le cas présent le mot " Moi " qui est étranger à la statue; d'où l'ignorance totale de son Moi [81].

Bonnet résout le problème de l'existence simultanée dans l'âme de diverses sensations ou idées par son approche de la réminiscence et de la personnalité. Sans la réminiscence, la même sensation dans l'âme "paraîtrait aussi nouvelle que si elle ne lui eût jamais été présente » [139-40]. Plusieurs sensations doivent pouvoir coexister dans l'âme. La réminiscence organise ce qui ne serait sinon qu'une succession désordonnée de sensations disparates en un Moi unifié sans lequel il n'y aurait point de personnalité [140]. Par conséquent, une "perte totale de la Mémoire, emporterait donc la destruction de la Personalité" [458]. L'acquisition ou la perte des idées, qui sont des processus normaux dans l'économie de notre être, ne «dénaturent » pas pour autant l'être; chacun d'eux "ne fer[a] que rendre la Personalité plus ou moins composée » [460].

Dans la mécanique de ses opérations, l'imagination procède de même que la mémoire à laquelle Bonnet l'identifie presque [134]. À l'instar de la mémoire, la faculté d'imagination reproduit pour l'âme les idées d'objets absents [155]. Même s'il commence par une affirmation métaphysique, Bonnet s'empresse immanquablement $d^{\prime}$ impliquer le physique, alliant par ce moyen le mental au sensoriel et renforçant sa position sensualiste. Cet enchaînement d'étapes physiologiques qui mène du corps à l'âme mérite d'être repris. Les idées correspondent aux mouvements des fibres sensorielles au sein d'un organe, cela est applicable au sens olfactif qui fait appel à des fibres spécialisées pour détecter le jeu des corpuscules émanant de l'objet, ici une rose ou un œillet $[47,52,155]$. L'imagination fonctionne en demandant au cerveau de répéter les mêmes mouvements initiaux. Dans le cas d'une idée composée qui émane de la confluence soit de deux ou plusieurs ordres de fibres $d^{\prime}$ un même sens, soit d'ordres de deux ou plusieurs sens affectés simultanément, l'imagination la reproduit grâce à " une communication secrette entre les différens Ordres de Fibres qui concourent à la production de cette Idée " [152, 156]. L'ordre dans lequel se succèdent initialement les idées simples ou concrètes influencera et déterminera bien entendu la manière dont l'imagination 
les reproduira [157]. Quelles que soient les fluctuations des produits de l'imagination et de la mémoire - nous imaginons ou nous rappelons plus ou moins au fil du temps dans l'économie de notre être - suffisamment de continuité est néanmoins préservée dans ces facultés pour nous donner le sentiment de notre personnalité.

Bien que la statue éprouve d'abord une impression de plaisir dans les degrés successifs de sa première sensation, ce phénomène devient "plus saillant » lorsque la statue a connu successivement deux sensations différentes [33-34]. Le plaisir et la douleur dérivent tous deux de la comparaison entre deux états distincts. Pour renforcer cette impression particulière de bien-être, Bonnet va même jusqu'à créer les néologismes "mieux-être » et «moins-bien-être » [33, 84]. Des implications grammaticales et économiques sont réunies dans ces termes qui évoquent très à propos les états changeants de notre être. Du point de vue grammatical, c'est le comparatif exprimé effectivement par les mots "mieux" ou "moins" qui reproduit le mieux les additions et soustractions appliquées à l'économie de notre être dont Bonnet tente tout au long de son ouvrage d'établir la nature davantage physique que métaphysique. La grammaire et plus particulièrement l'économie fournissent au sujet de notre être des explications moins éphémères, et donc pour Bonnet davantage plausibles que ne le fait la métaphysique.

Bonnet définit le plaisir et la douleur en termes de mouvement qui sert également de catalyseur à la sensation": "Nous sçavons seulement que toute Sensation tient à un mouvement, \& qu'un mouvement plus ou moins fort, plus ou moins acceleré fait naître la Douleur ou le Plaisir» [87]. Plus le mouvement est marqué, plus le plaisir qui en dérive est vif. Il y a pourtant des limites à l'intensité de mouvement qui peut être tolérée. Ce qui s'annonce comme une expérience agréable peut s'avérer pénible. Bonnet, on s'y attend, recourt au physique pour décrire le processus: «la même Fibre qui produit le Plaisir lorsque ses vibrations sont accélerées dans un certain degré, fait naître la douleur lorsque ces vibrations sont accélerées au point de séparer trop les unes des autres les Molécules de la Fibre» [87].

Le plaisir et la douleur jouent un rôle important dans l'économie de la statue. À mesure que son être se développe grâce à l'intervention d'organes nouveaux, elle en vient surtout à apprécier son existence au travers d'activités qui lui procurent 
du plaisir [453-55]. La statue est heureuse de sentir et d'agir, du moment qu'elle peut le faire agréablement [ibid.]. Elle enrichit son existence en multipliant les successions de sensations agréables [456-57]. Si, d'une part, l'accumulation de sensations agréables favorise la conscience du Moi, la douleur, d'autre part, "avertit l'Individu de ce qui touche à la destruction de son Être" [89]. Le plaisir et la douleur se révèlent donc être des facteurs décisifs non seulement du développement mais encore de la préservation de l'économie de notre être. Leurs agents, les sensations, bien que guidant peut-être parfois l'homme vers la perfection, ont pour fonction première sa conservation $[E P$ 48; EA 316].

La réaction de l'âme au plaisir, qui lui fait préférer une sensation plutôt qu'une autre, est appelée attention [33-34, 101-02]. Dans la suite des sensations sur lesquelles l'âme porte son attention, il ne faut pas non plus perdre de vue les deux formes possibles de sensations qui sont également applicables à l'attention: sensations radicalement différentes ou "différens degrés dans la même Sensation " [33]. Mais c'est à dessein que Bonnet reporte la «discussion délicate » à propos de l'attention, du plaisir et du désir jusqu'à ce que la statue connaisse deux sensations différentes et que le lecteur soit "placé assez avantageusement » pour saisir les nombreuses nuances du processus [34].

Bonnet dirige finalement le débat sur l'attention vers la question suivante: «que résulte-t-il dans l'Âme de nôtre Statuë du plus ou du moins de Plaisir que deux Sensations différentes lui font éprouver? " [103]. La réponse simple, énoncée à la suite d'un important préambule, est la notion de préférence, fondamentale à l'attention. Le facteur décisif en ce qui concerne l'attention consiste donc en la préférence que l'âme donne à la sensation qui l'affecte le "plus agréablement " [ibid.]. La réponse de Bonnet met l'accent, ainsi que le fait la question, sur la quantité et la qualité des deux sensations dont la statue fait l'expérience: l'odeur d'une rose et celle d'un œillet. Elle insiste davantage sur l'importance de l'activité impliquée dans la préférence de la statue: "Cette préférence que la Statuë donne à la Sensation qui lui plaît le plus, est une action que la Statuë exerce sur cette Sensation. Préférer n'est pas Sentir, c'est se déterminer, c'est agir. La préférence ne peut être une modification de la Faculté de sentir: les modifications de cette Faculté ne sont que des Sensations, \& des degrés de Sensations " [104, 279]. Il est clair que l'attention n'est pas une sensation, mais à quel 
moment intervient-elle ${ }^{10}$ ? L'étude de Bonnet mène précisément à cette question, qui, en dépit du fait qu'elle ne soit jamais véritablement posée, reçoit pourtant une réponse: "L'Attention est donc une modification de l'Activité de l'Âme; ou, pour m'exprimer en d'autres termes, elle est un certain exercice de la Force motrice de l'Âme sur les Fibres de son Cerveau» [105].

À première vue, une telle explication paraît contredire la position sensualiste de Bonnet concernant la subordination des activités de l'âme à la sensibilité [87, 99]. Bonnet semble insinuer que dans l'acte d'attention l'âme agit indépendamment de la faculté de sentir. L'examen minutieux de son argument révèle toutefois la cohérence de son sensualisme. Chez Bonnet, l'équivoque dans l'analyse des actions de l'âme sur le corps est levée par son hypothèse que "l'Âme modifie l'état actuel de son Corps" [99]. Mais cet état actuel qui précède une action est une perception ou sensation d'un certain degré de plaisir ou de douleur: "Un Être Sentant ne peut être déterminé à agir qu'en vertu d'une Perception, ou d'une Sensation agréable, ou désagréable dont il est affecté " [101]. Lorsque l'âme agit, elle le fait uniquement en réponse aux sensations déjà présentes dans le corps et $n^{\prime}$ agit pas indépendamment d'elles. En ce sens, son activité est indéniablement subordonnée à la sensibilité.

Bonnet sauvegarde son sensualisme en même temps qu'il fait valoir le rôle actif de l'âme. Parmi les trois grands sensualistes, Condillac, Helvétius et Bonnet, ce dernier est le seul qui s'attache sérieusement au problème de la passivité de l'esprit inhérent à la thèse épistémologique. Sans abandonner sa position fondamentalement sensualiste qui lie l'esprit et le corps en une union indissoluble, Bonnet $s^{\prime}$ élève contre le concept d'un être humain tout juste capable de refléter, tel un miroir, l'image d'objet extérieurs et qui éprouve et rappelle les sensations sur un plan strictement physique indépendamment de l'âme $[86,104]$. La nature active de l'âme se manifeste tout particulièrement dans l'acte d'attention ${ }^{11}$.

Son étude d'une faculté cognitive aussi importante que l'attention est enchâssé, on s'y attendra, dans une analyse de l'économie de notre être. Ainsi que je l'ai souligné tout à l'heure, la question sur l'attention est posée en termes quantitatifs: la statue tire "plus ou moins" de plaisir des sensations olfactives qui résultent de son interaction avec la rose et l'œillet. Bonnet incite même le lecteur à faire l'expérience de l'économie de 
son être en fixant des yeux un quelconque objet puis en les détournant. Alors qu'il "affaibli[ $\mathrm{t}]$ " ou diminue l'impression des objets environnants quand il s'en détourne et y fait moins attention, en accordant plus d'attention à un seul objet, par contre, il "tend" ou intensifie son regard sur lui [105].

L'explication mécanique de l'attention étaye et corrobore l'idée d'une économie dynamique à l'œuvre dans notre être. L'insistance de Bonnet sur l'aspect physique de l'attention met d'autant plus en valeur sa nature «économique». Dans l'acte d'attention, l'âme n'agit pas sur la sensation proprement dite, " puisque cette Sensation n'est que l'Âme elle-même modifiée d'une certaine maniére, " mais "sur les Fibres dont le mouvement produit la Sensation" [106]. Le lecteur doit reconnaître ce que quiconque tire pour leçon des «Faits » quotidiens de l'expérience sensorielle: "que l'Attention augmente l'intensité des mouvemens imprimés par les Objets » [107]. Une certaine fluctuation dans la précision des perceptions est accompagnée d'une fluctuation du fluide nerveux. Bonnet écrit: " À mesure que la Perception de l'Objet devient plus vive par l'Attention les Perceptions des Objets voisins s'affaiblissent " [110]. Si l'intensité d'une perception s'accroit, celle d'une autre diminue. Sur le plan physiologique, l'attention accélère le mouvement des fibres concernées, qui par conséquent consomment une plus grande quantité d'esprits ou de fluide aux dépens des fibres avoisinantes [110-11]. À proprement parler, ce qu'une fibre gagne en fluide une autre la perd. Les descriptions que fait Bonnet des opérations mentales et de leurs activités physiques concomitantes visent à faire naître une vivante image de l'économie de notre être.

En relation étroite avec la faculté d'attention qui établit la nature active de l'âme, se trouvent les facultés de volonté et de liberté. La statue commence déjà à les exploiter dès la seconde sensation [114]. À l'instar de l'attention, la volonté est basée sur une préférence accordée par l'être à ce qui lui fait du bien plutôt que du mal: "voulork est cet acte d'un Être sentant ou intelligent; par lequel il préfére entre plusieurs maniéres d'être celle qui lui procure le plus de bien, ou le moins de mal »[114-15, 278]. Puisque la statue a besoin de connaître et de sentir plus d'une sensation ou d'états d'être avant de manifester une préférence, la "Volonté est donc subordonnée à la Faculté de sentir, ou de connoitre" [115]. Le motif indispensable pour vouloir produire une action est «toûjours une Sensation, une Idée » [287]. 
Bonnet élargit son analyse sensualiste de la volonté lorsqu'il déclare que la raison nécessaire à toute volonté peut se trouver «dans l'ebranlement des Fibres sensibles, d'où résulte cette Modification de la Faculté de Sentir, qu'on nomme Sensation, Idée " [299]. Dans les deux citations précédentes, Bonnet place sensations en apposition à idées, ainsi qu'il avait antérieurement mis en parallèle connaître et sentir en parlant de la subordination de la volonté, et qu'il le fait à nouveau à la même page dans le passage suivant: "La Volonté suppose donc la connoissance ou le Sentiment de différentes maniéres d'être " [115]. Les deux termes ne sont de toute évidence pas exactement synonymes, mais dans l'esprit des sensualistes, et selon eux dans nos esprits, il existe un rapport continu et donc causal entre ces opérations. Le refrain sensualiste selon lequel tout savoir provient des sens est repris maintes fois au cours de ces pages. Qui plus est, les sens et la sensibilité en viennent à concerner et façonner l'ensemble des facultés.

Lorsque Bonnet définit ainsi la faculté de liberté: "la Faculté par laquelle l'Âme exécute sa Volonté " [116] ou "le Pouvoir d'exécuter sa Volonté» [287], il la soumet chaque fois à la même hiérarchie dans laquelle vont s'ordonner toutes les autres facultés. Puisque de nouveaux éléments y ont été introduits, l'ordre mérite d'être repris: "la Liberté est subordonnée à la Volonté comme la Volonté l'est à la Faculté de Sentir " qui, à son tour, est subordonnée à "l'action des Organes » résultant de "celle des Objets" [116]. Les pouvoirs des facultés supérieures sont systématiquement assujettis à ceux de la sensibilité; chez les êtres sentants, l'action dépend des qualités agréables ou désagréables des sensations [430]. L'exemple fourni par Bonnet pour prouver la théorie sensualiste selon laquelle la sensibilité est le centre de rayonnement des activités de la statue est révélateur. $\mathrm{Si}$ son âme choisit de passer directement d'une sensation $A$ à une sensation $F$ selon une séquence dont la statue a déjà fait l'expérience, toutes les sensations intermédiaires doivent "lui [être] moins agréables" [ibid.]. Plus loin dans l'essai, Bonnet résume la même leçon en une phrase concise: "La Loi du Plaisir, est donc la Loi de la Volonté " [299]. Bien qu'il prenne ici en considération les plaisirs moraux éprouvés par les êtres doués de réflexion, l'autorité de la sensibilité dans la détermination des actions et volontés humaines reste intacte. 
À propos de la liberté, Bonnet désapprouve la définition que Condillac donne de cette faculté. Alors que chez Condillac la liberté est "le Pouvoir de faire ce qu'on ne fait pas" ou celui "de ne pas faire ce qu'on fait », chez Bonnet, son essence réside dans l'action et «le Pouvoir de faire ce que l'on fait « [122-23]. Bonnet propose un exemple emprunté à la Dissertation sur la liberté de Condillac. Dans l'un des passages typiquement rationalistes, Condillac insiste sur la délibération ou capacité de délibérer qui doit précéder tout acte libre. En réalité, Bonnet en tant que sensualiste s'engage plus fermement que Condillac sur cette question et souligne le libre choix impliqué chez l'être dans l'acte de faire "ce qui lui plaît " [125]. Pour Bonnet, un acte qui s'accommode à notre volonté, principalement déterminée par notre sensibilité, est toujours un acte libre [280-81]. La liberté réside en l'exécution de la volonté: “On est donc libre toutes les fois que l'on fait ce que l'on veut» [284-85]. Dans sa conception de la volonté, Bonnet maintient donc encore le rôle central des sens.

Il devient aisé de discerner aussi le désir lorsque la statue a deux sensations à comparer. Lorsqu'elle ne sent qu'une seule et même odeur de rose, la statue représente le niveau le plus bas sur l'échelle de l'animalité et n'éprouve aucun désir qui "suppose la connoissance d'un état différent de l'état actuel, \& qu'on lui compare» [31]. Une prise de conscience du désir nécessite donc une progression dans l'échelle ou la chaîne des êtres, ce qui met en œuvre certaines des facultés grâce auxquelles les êtres humains peuvent se différencier des autres matières et des autres formes de vie. La comparaison entre différents degrés, ne serait-ce que d'une seule et même sensation, nous fait éprouver, par rapport à l'expérience antérieure, une impression de mieux-être ou de moins-bien-être. Plaisir, attention et désir se rejoignent en cet instant qui marque la naissance d'un nouvel être: «La Connaissance d'un mieuxêtre est inséparable du désir de la continuation du mieux-être; \& l'effet de ce désir est l'Attention" [33]. Le lecteur comprendra l'importance d'une convergence aussi riche de facultés et d'activités à un stade similaire mais ultérieur dans le développement de la statue, lorsqu'elle aura éprouvé deux sensations distinctes. Bonnet ne peut pourtant pas s'empêcher de faire allusion à l'impressionnant éventail de capacités cognitives qui restent à découvrir à la statue: «je ne pouvois me dispenser d'indiquer tout ce qui étoit renfermé dans ce premier état de nôtre Statuë» [34]. 
Ce premier état est indubitablement pour Bonnet une phase féconde. L'état primitif de la statue donnera en effet naissance aux facultés supérieures de la même façon que les germes contenus dans nos corps donneront naissance à des formes d'être plus parfaites lors de la résurrection des morts, selon la vision élaborée que se fait Bonnet de l'au-delà. Bonnet y réunit des éléments de religions orientales et occidentales; après avoir mentionné la résurrection des morts, il progresse tard dans l'essai vers une théorie de la réincarnation: "Une Saine Philosophie nous apprend à penser, qu'il n'est point dans la Nature de vraye Génération; mais, que les Corps qui nous paroissent être engendrés, ne font que se développer, parce qu'ils existaient déja tout formés en petit, dans des Germes » [485]. De ce qui à l'origine apparaît trompeusement petit et simple jaillit à un stade plus avancé du développement une forme glorieuse en puissance.

En tant qu'entomologiste, Bonnet était bien entendu familiarisé avec les changements phénoménaux subis par la chenille au cours de sa métamorphose en papillon. Il n'hésite pas à citer I'exemple de la chenille pour illustrer la marche progressive de la nature [481]. On commence de ce fait à comprendre le dynamisme de sa méthode qui fait coïncider la continuité du monde naturel avec celle du processus cognitif. Ce que dit Bonnet à propos de la chenille est applicable à son étude de l'âme: "La marche de la Nature ne se fait point par Sauts. Elle prépare de loin, \& dans une obscurité impénétrable, les Productions qu'elle expose ensuite au grand jour " [130, 481]. Comme la chenille, la statue possède les germes de sa perfection, si invisibles soient-ils aux yeux inexperts. Dans sa propre anticipation des activités prospectives de l'âme, Bonnet agit, pour ainsi dire, comme un futur parent qui ne peut s'empêcher d'imaginer et de faire partager à ses amis (ici les lecteurs) ce que nous réserve l'avenir.

Par la dégradation du mouvement dans les fibres sensibles et par extension dans l'économie de notre être, Bonnet établit enfin le lien entre désir, plaisir et attention qu'il n'avait fait que suggérer précédemment dans un élan d'intuition. Par "dégradation" Bonnet entend la diminution graduelle ou l'affaiblissement du mouvement que les objets occasionnent sur les fibres sensitives d'un organe [32-33; 129]. Toute sensation a ses répercussions, la plus remarquable étant peut-être celle dont on fait l'expérience en fermant les yeux après avoir fixé le soleil [EP 69-70]. Après que la statue a senti l'odeur d'œillet 
qu'elle préfère à celle de la rose en raison du plaisir accru que lui procure cette nouvelle sensation, son attention est engagée. Par conséquent, elle suit et ressent d'autant plus profondément la dégradation du mouvement qui était à l'origine de la sensation [131-32]. Le désir résulte donc de l'affaiblissement graduel mais perceptible d'une sensation qui a procuré du plaisir; il augmente à mesure que la sensation agréable diminue [132]. Dans l'économie de notre être, l'intensité du désir s'accroît à mesure que s'accroît la différence ou l'écart entre la situation actuelle et un moment de bien-être passé auquel on accorde la préférence et que la mémoire rappelle à l'esprit [132-33]. L'imagination ne sert bien entendu qu'à attiser le désir en créant un simulacre de la sensation agréable, qui, pour autant qu'il ressemble au moment original désiré, $\mathrm{s}^{\prime}$ en distingue tout de même sensiblement [134-35]. Pour Bonnet, le désir constitue en fin de compte l'activité la plus fréquente chez l'être sentant: "plus on [sent et donc] connoît, plus l'on désire" [495]. Du point de vue de son sensualisme, sentir, savoir et désirer sont en étroite corrélation, comme le fait valoir l'analyse des idées.

Bien que Bonnet "abandonne" [145] momentanément le sujet de la statue lorsqu'il se tourne vers celui de l'évolution des idées, son point de départ reste le même, à savoir les deux premières sensations de la statue. Sa méthode analytique l'oblige à "épuise[r] tout ce qui [en] découle nécessairement " [144]. En ce qui concerne la logique sensualiste, bien peu de choses coulent de source dans la façon dont les idées découlent des sensations: "Toutes nos idées dérivent donc originairement des sens " [13]. Bonnet définit d'abord grossièrement une idée comme " toute manière d'être de l'Âme dont elle a la conscience ou le sentiment " [13-14]. Toutefois, dans sa théorie générale des idées, il raffine la définition précédente qui s'appliquait à "l'Origine de toute Idée " en faisant remarquer que:

les maniéres d'être de l'Âme varient comme les degrés de sa Perfection. Le mot Idée reçoit donc différentes déterminations suivant les maniéres d'être que l'Âme revêt.

Tantôt il n'exprime que de pures Sensations tantôt il désigne des Notions. Il s'applique ainsi au Sentiment \& à la Réflexion. [145].

Bonnet adopte dans ce passage un ton manifestement lockéen qu'il reprendra plus tard lorsqu'il affirmera de façon étonnamment vague et désinvolte: «l'on dit que nos Idées ont deux sources, 
les Sens \& la Réflexion" [173]. Ce que "l'on dit" (on étant évidemment une référence impersonnelle surtout pour Locke) ne correspond cependant pas à ce que dit Bonnet ${ }^{12}$.

La lecture des chapitres XIV à XVI donne de prime abord l'impression qu'en abandonnant momentanément la statue, Bonnet abandonne aussi son sensualisme. Cette entorse dans la continuité de son discours sur la statue semble succéder à une entorse dans ce qui a été jusqu'ici chez Bonnet une ferme adhérence aux doctrines sensualistes. Certes, "l'état d'un Être purement Sentant différe beaucoup de l'état d'un Être intelligent » ainsi qu'il le fait remarquer plus tard en justifiant cette section de l'essai [355]. Mais en fin de compte, Bonnet ne sera pas plus disposé à accepter une rupture dans la chaîne du processus cognitif qu'il ne le sera à en accepter une dans la chaîne des êtres. Il reste plutôt fervent partisan de sa méthode analytique qui insiste sur la continuité avant tout ${ }^{13}$. Le sensualisme de Bonnet découle précisément de sa méthode analytique qui garantit la continuité. Le continuum de la nature qui, postule Bonnet, englobe les minéraux et l'homme, est homologue à celui de la cognition qui englobe d'un même élan sensations et idées. Les points transitoires essentiels qui rallient les sensations aux idées sont appelés les idées sensibles. Pour appréhender la chaîne cognitive de Bonnet, nous devons retrouver les éléments qui partent de ces points ou y aboutissent.

D'après Bonnet, les idées sensibles, composées d'idées simples et d'idées concrètes, proviennent de "l'Action des Objets sur les Sens» [152]. Il les oppose à celles «dont la formation tient à quelque Opération de l'Esprit " [ibid. $]^{14}$. Les idées simples ne peuvent être décomposées par l'âme en d'autres idées, "parce qu'elles répondent à une Impression qui est une \& simple " [148]. Ce sont, par exemple, des sensations qui ont trait à toutes les formes de perception (comme les odeurs, les goûts, les sons, les couleurs, le froid et la chaleur); perceptions de l'étendue, de la solidité, de la force d'inertie et du mouvement; ainsi que la perception des effets de forces physiques et intellectuelles irréductibles [148-50]. Bonnet distingue les sensations des perceptions par le degré d'ébranlement des fibres sensibles qui n'est pas aussi marqué pour les secondes que pour les premières. Une perception qui dénote "la simple appréhension de l'Objet " peut devenir une sensation si toutefois l'ébranlement des fibres est suffisamment accru pour déclencher le plaisir ou la douleur qui accompagne toujours une sensation [14546]. 
Les idées concrètes ou composées diffèrent des idées simples par le nombre d'ordres de fibres affectés. Elles interviennent dans les cas où "deux ou plusieurs Ordres de Fibres d'un même Sens, ou que des Ordres de Fibres de deux ou de plusieurs Sens sont ébranlés à la fois par un Objet » [152]. Se rangent dans cette catégorie d'idées tous les corps que nous percevons dans notre environnement [ibid.]. Grâce au pouvoir d'abstraction sensorielle qui est "un Acte de l'Attention ", l'âme peut décomposer ces idées [153-54]. À cet égard, l'activité de l'âme pourrait sembler indiquer un processus totalement rationnel, dénué de tout contact avec les sens, si l'on faisait abstraction du fait que Bonnet subordonne toutes ses activités à la sensibilité ${ }^{15}$. En effet, afin de préserver la cohérence au sein de son argument, Bonnet se doit d'affirmer que: "l'Idée abstraite n'est qu'une Idée sensible détachée par l'Attention du Tout dont elle faisoit partie» [154].

Lorsque Bonnet fait progressivement évoluer sa discussion du langage vers des abstractions complexes apparemment toutes aussi étrangères à l'univers sensible, il souligne néanmoins le rapport avec les sens: "Les Idées que nous recevons par les Sens, nous les revêtons de Signes, ou de Termes qui les représentent " [158]. Bien que les objets puissent être absents ou ne plus être accessibles à nos sens, les signes ont une "présence" physique que les yeux ou les oreilles sont capables d'intercepter et qui " réveille l'Idée qui leur a été attachée » [159]. Les signes eux-mêmes servent pour ainsi dire d'objets sensibles qui rappellent d'autres objets à l'esprit. En vertu de ce pouvoir, ils "multiplie[nt] les Liens qui unissent nos Idées, " augmentant le nombre des "Liaisons naturelles qui résultent de la maniére dont [les idées] ont été excitées par les Objets, \& de l'Analogie des Objets entr-eux » [160]. De plus, les signes contribuent à créer une nouvelle forme d'abstraction qui, en décomposant et séparant les idées sensibles, conduit à son tour à une plus vaste généralisation et classification des idées [161-62]. Le mouvement s'éloigne de la nature et de ses objets pour s'orienter vers un système artificiel et arbitraire de signes: "Ces Abstractions par lesquelles l'Âme généralise ses Idées tiennent moins à ce qui est dans la Nature, que n'y tiennent les Abstractions sensibles. À mesure que l'Abstraction est poussée plus loin par l'intervention des Signes, les Idées qui en naissent s'éloignent d'avantage des Idées purement sensibles » [163-64].

La logique sensualiste qui, à maintes reprises, cède du terrain pour refaire triomphalement surface, est mise une dernière 
fois à l'épreuve lorsqu'est abordé le sujet des abstractions intellectuelles génératrices des notions et de la réflexion. Les abstractions intellectuelles donnent naissance aux idées générales ou notions telles celle du "Chêne en général " qui bien sûr n'existe pas dans la nature [165]. La rupture entre le corps et l'esprit se fait apparente lorsque Bonnet distingue d'un côté l'interaction du monde extérieur et des sens - une des principales activités de l'âme - et d'un autre côté l'activité de l'esprit: "La Notion n'est donc pas une Perception: elle ne résulte pas simplement de l'Action de l'Objet sur les Sens; elle suppose encore une opération de l'Esprit sur cette Action " [ibid.]. Pour un sensualiste comme Bonnet qui s'acharne à prouver la nature mixte ou l'indivisibilité du corps, de l'âme et de l'esprit, ceci représente un point extrêmement délicat où l'union entre ces éléments constituants peut réussir ou manquer ${ }^{16}$. Bonnet n'utilise plus alors l'âme mais l'esprit pour indiquer les mécanismes internes responsables de la production de ces nouvelles idées ou notions. Du fait que la hiérarchie de Bonnet subordonne uniquement les activités de l'âme à la sensibilité, il apparaît que l'esprit pourrait agir indépendamment des sens pour générer les abstractions intellectuelles appelées notions.

Ce $n^{\prime}$ est pas avant le chapitre suivant, quelques huit pages plus loin dans l'essai, que Bonnet réaffirme son sensualisme en clarifiant l'interdépendance du corps, de l'âme et de l'esprit. $\mathrm{Au}$ tout début de ce chapitre, il déclare brusquement (étant donné le hiatus de huit pages) et sans ambages: "C'est donc en opérant sur les Idées sensibles que l'Esprit acquiert des Notions. Cette opération porte le nom de Réflexion " [173]. Les renvois spécifiques dans le texte de Bonnet au passage cité plus haut nous permettent d'aborder la partie précédente dans une perspective nouvelle ${ }^{17}$. Les expressions "pas simplement" et "encore " (encore pris dans le sens de "en plus " ou «toujours") confirment plus qu'elles ne dénigrent le rôle des sens, même en ce qui concerne les facultés cognitives supérieures, et indiquent un niveau d'activité qui coïncide partiellement avec les sens plutôt que d'en être séparé. La réflexion ou l'opération par laquelle l'esprit acquiert des notions sous-entend l'existence, à un stade antérieur, d'idées sensibles.

Bonnet en revient aux sens, par la voie des idées sensibles, afin de montrer ce qu'est la réflexion qui, selon ses termes, résulte: «de l'Attention que l'Esprit donne aux Idées sensibles, qu'il compare, \& qu'il revêt de Signes, ou de Termes qui les représentent " [174]. L'esprit proprement dit ne peut s'élever 
d'un bond du néant à des idées hautement abstraites ou «réfléchies". Les sens sont impliqués à chacun des niveaux intermédiaires y compris les plus élevés. Si l'on en croit Bonnet, l'image même de Dieu est basée sur les sens: «Nos Idées les plus abstraites, les plus spiritualisées, si je puis employer ce mot, dérivent donc des Idées sensibles, comme de leur source naturelle. L'Idée de DIEU, par exemple, la plus spiritualisée de toutes nos Idées, tient manifestement aux Sens »[174-75].

Afin de résoudre la question des sources de nos idées, Bonnet se place dans la lignée de Locke en les attribuant aux sens et à la réflexion. Mais dans le schéma sensualiste des choses que trace Bonnet, la réflexion ne peut être entièrement isolée des sens. Malgré certaines hésitations passagères de la part de Bonnet, la continuité entre sens et réflexion devient nette à mesure qu'il avance dans son analyse. Quoique Bonnet implique une relation entre idées "concrètes " et idées "générales " dans le passage sur le langage des signes, au chapitre $X V$, ce n'est qu'au chapitre suivant qu'il en discute explicitement. Il ne se borne pas alors à de franches affirmations telles que "Nous l'avons vû: l'Esprit tire ses Notions des Idées sensibles" mais il renvoie également à des passages du chapitre précédent qui lui ont permis de construire son argument [184].

En vertu du rapport continu qu'il établit entre les sens et les idées, Bonnet suggère aussi l'existence d'un lien entre diverses formes de vie, à savoir, l'homme et l'animal. Ces deux classes d'êtres sont en effet extrêmement proches l'une de l'autre, ainsi que le démontre Bonnet avec sa statue. La première forme de personnalité qu'elle développe s'applique "également aux Animaux, \& même à ceux qui sont le moins élevés dans $l^{\prime}$ Échelle " [81]. Bonnet nous rappelle même que l'état actuel et primitif de sa statue, bien que celle-ci soit de constitution humaine [8, 208], "représente celui d'un Animal qui n'auroit qu'un seul Sens, \& dont tous les besoins \& tous les mouvements seroient rélatifs à l'exercice de ce Sens " [500]. Si la sensibilité des animaux qui, comme nous, ont des idées sensibles, les place au-dessus des plantes dans l'échelle des êtres, l'homme se distingue des animaux par sa capacité à généraliser des idées dans l'abstrait au moyen de signes artificiels [180, 500, 523]. Bonnet n'exclut pas pour autant la possibilité que les animaux ou les êtres qui leur sont égaux puissent un jour développer leur entendement [EP 178; EA 180-81]. Sa chaîne des êtres et son concept de "germes" rendent possible cette progression sur l'échelle. Fort de cette explication sur 
l'évolution des idées, Bonnet ne franchit finalement le pas qui sépare un être purement sentant d'un être intelligent que pour montrer le lien qui les unit en dépit de leurs différences majeures ${ }^{18}$.

Pour conclure, en se limitant à deux sensations chez sa statue, Bonnet attire notre attention sur la nature dynamique et successive de notre être. Grâce à elles, il peut non seulement expliquer les facultés de l'âme et la formation des idées, mais encore apporter la preuve de son profond attachement au sensualisme et à sa méthode analytique qui sont inséparables dans son ouvrage. Dans l'enchaînement d'idées que Bonnet s'évertue à obtenir grâce à une analyse dont la pierre angulaire est la succession, une idée basée sur des faits mène à une autre de façon à former une chaîne. Sa formation professionnelle de scientifique dispose favorablement Bonnet envers le monde physique et la fiabilité des informations qu'il procure. Il ne sépare jamais vraiment le scientifique ou le physique de la métaphysique; d'où son choix de parler d'une économie plutôt que $d^{\prime}$ 'une métaphysique de notre être: "En un mot, la Science de l'Âme, comme celle des Corps, repose également sur l'Observation \& l'Expérience" qui étudie la nature et non les abstractions [xviI]. A l'image de la nature, la forme d'analyse choisie par Bonnet ne néglige aucun maillon de la chaîne pas plus qu'elle ne procède par bonds. Elle progresse de manière ininterrompue depuis le monde observable de l'expérience sensorielle dans laquelle elle puise son autorité. Sa méthode met en parallèle une vision continue de la nature et une vision similaire de la connaissance humaine. L'Échelle des Êtres trouve sa contrepartie épistémologique dans les étapes successives du processus cognitif. En suivant "le Fil analytique " [Ix] qu'il prétend innocemment observer avec ses yeux, se laissant guider par l'enseignement de l'expérience, Bonnet retrouve naturellement la source des idées dans les sens. Pour un observateur qui, comme Bonnet, insiste sur la primauté du monde physique que l'on appréhende uniquement par les sens, l'analyse appliquée à l'épistémologie conduit inévitablement au sensualisme. Sa méthode corrobore donc essentiellement son message. Dans l'économie de notre être, la "dépendance réciproque " [355] des facultés est à la fois démontrée et renforcée par la méthode analytique de Bonnet, ainsi que l'est, en fin de compte, la continuité entre les sensations et les idées, et entre le corps et l'esprit.

Romance Languages and Litterature

Hamilton College, Clinton, New-York 


\section{NOTES}

1. Charles Bonnet, Essai analytique sur les facultés de l'âme, (Copenhague, 1760); réédition Hildesheim et New York: Olms, 1973, pp. 10-11. Sauf indications contraires, toutes les références de pages se rapportent à cet ouvrage - rappelé par la suite sous l'abréviation $E A$, lorsque cela s'avérera nécessaire par mesure de clarté - et sont faites au fil du texte, ainsi qu'il en est des références à ses autres cuvres. Les majuscules, la ponctuation et les italiques dans les citations sont de Bonnet.

Pour une étude comparative des statues de Condillac et de Bonnet, voir Albert Lemoine, Charles Bonnet de Genève, Philosophe et naturaliste (Paris: A. Durand, 1850), pp. 116-47. Lemoine félicite Bonnet de sa méthode patiente et rigoureuse mais lui reproche de n'avoir pas amené sa statue, comme le fait Condillac, jusqu'au niveau d'un homme dont tous les sens sont opérationnels.

2. Charles Bonnet, Essai de psychologie; ou considérations sur les opérations de l'âme, sur l'habitude et sur l'éducation (Londres, 1755); Hildesheim et New York: Olms, 1978, voir notamment pp. 153-54. Les références empruntées à cette oeuvre sont indiquées par l'abbréviation EP. Rédigé sur un ton très timide, l'Essai de psychologie paraphrase les arguments d'autres philosophes et fait de nombreuses conjectures et propositions hypothétiques. Apparemment, Bonnet n'a pas encore trouvé ni sa voix authentiqu ni sa propre méthode. En contraste frappant avec le rythme de l'Essai analytique dont Bonnet consacre la plupart des pages aux deux sensations, cet ouvrage progresse rapidement, en tout juste vingt-sept pages, de la conception du fœetus jusqu'aux idées du bien et du mal, pp. 5-32.

3. Charles Bonnet, Principes philosophiques sur la cause première et sur son effet (Londres, 1755); réédition, Hildesheim et New York: Olms,1978, pp. 296-98. À l'édition originale de 1754 et à celle de 1755 de l'Essai de Psychologie, Bonnet ajouta cette autre partie distincte. Cet ouvrage sera mentionné ci-après par l'abréviation $P P$.

4. La méthode de Bonnet est ici comparable à celle qu'il approuve dans l'Essai de psychologie [244] concernant l'éducation du jeune Architas doté du sens de la mécanique. Le mouvement du connu vers l'inconnu est doublé d'un mouvement du simple vers le complexe. Le processus par lequel nous apprenons doit toutefois être parfois inversé pour expliquer l'état actuel de notre être. En décrivant sa méthode analytique pour la première fois, Bonnet utilise l'expression "du composé au simple " [EA 1]. Mais lorsqu'il développe ce qu'il entend par là [2] et récapitule sa méthode [65], il est clair qu'il procède du simple au composé.

5. Continuité, gradation et plénitude sont les principes sous-jacents à la vision de l'univers comme une Chaîne des Etres, vision qui 
prévaut au dix-huitième siècle. Du fait de son insistance sur la continuité de la Chaîne, Bonnet contribue grandement à renforcer cette vision. Voir Arthur O. Lovejoy, The Great Chain of Being: $A$ Study of the History of an Idea (1936); réédition Cambridge, Mass. et Londres: Harvard University Press, 1978, pp. 183-287, notamment pp. 230-31, 235, 275, 283. Lovejoy cite La Contemplation de la nature de Bonnet (1764-65) et remarque que l'auteur est prompt à associer l'homme et le singe dans la Chaîne continue des Êtres.

6. Bien qu'au chapitre XXI et au-delà il continue à présenter d'autres sensations olfactives à la statue et finit par lui « ouvrir » les oreilles [507], sa méthode reste essentiellement inchangée. Son analyse approfondie de l'odorat " peut s'appliquer facilement aux autres sens $»[538]$.

7. Aux facultés de mémoire, imagination, volonté et liberté, j'ai ajouté attributs ou sentiments, personnalité, plaisir et douleur, et désir, du fait que Bonnet traite de ces facultés en ces termes ou bien les regroupe.

8. Ainsi que je suis sur le point de le démontrer, cette différence n'est pas une division discontinue.

9. C'est seulement plus loin dans l'Essai [145] que Bonnet reconnaît que les sensations entretiennent une relation particulière avec le plaisir et la douleur. Toute sensation est invariablement accompagnée de l'un ou de l'autre. Voir également les pages 96 et 97 suivantes.

10. Bonnet se borne à rechercher les " faits " qui consistent en grande partie à "connaître l'Ordre dans lequel les Choses se succédent " plutôt que leurs causes dont bon nombre nous restent inconnues [93-95].

11. La représentation que se fait Bonnet de l'âme en tant que force active remonte à Leibniz. Voir Georges Bonnet, Charles Bonnet (1720-1793), Paris: M. Lac, 1929, p. 87.

12. Dans sa thèse, Lemoine remarque les différentes manières dont Locke et Bonnet conçoivent la réflexion. Bonnet n'en fait qu'une forme supérieure de la sensibilité; alors que pour Locke, elle est " synonyme de la conscience et s'oppose à la sensation; la réflexion de Bonnet est synonyme de l'attention et s'oppose à l'exercise involontaire de la sensibilité et de l'intelligence ". Voir Albert Lemoine, Charles Bonnet de Genève, Philosophe et naturaliste, p. 116.

13. En dépit de sa vaste expérience avec les plantes et les animaux, Bonnet maintient la notion d'un continuum artificiel de la matière inorganique vers la matière organique. Voir Lorin Anderson, "Charles Bonnet's Taxonomy and Chain of Being », Journal of the History of Ideas, 37, 1976, 45-58. Anderson explique le "zèle » avec lequel Bonnet s'intéresse à la ressemblance entre l'homme et le singe dans une perspective qui ne situe plus les connections dans le 
temps, comme le fera l'évolutionnisme au dix-neuvième siècle, mais dans un « arrangement statique » [45-46; 54-55].

14. Il n'est pas inutile de répéter que les opérations de l'esprit, si différentes soient-elles de celles du corps n'en sont pas séparées selon le point de vue sensualiste de Bonnet. Bonnet n'explicite clairement ce sujet que plus tard dans son essai.

15. Voir ma discussion sur l'attention ci-dessus pp. 98-99.

16. Avec l'acquisition et la dégradation des sensations, l'économie de notre être s'apparente à l'économie de l'argument de Bonnet dans lequel les témoignages en faveur de la primauté des sens dans la détermination des idées sont d'abord accumulés puis dispersés parmi les diverses facultés. Le contenu et la forme se complètent.

17. Dans l'Essai analytique, Bonnet utilise un système élaboré de renvois numérotés, basé sur les diverses parties d'un chapitre donné. Un chiffre est placé au début de chaque partie et repris par la suite entre parenthèses pour renvoyer à la partie correspondante.

18. Selon Lester G. Crocker, dans l'intérêt que Bonnet porte au continuum corps-esprit, il suit la théorie de l'association d'idées de David Hartley. Voir son article sur Bonnet dans The Encyclopedia of Philosophy, New York et Londres: Macmillan and the Free Press, 1967, t. 1, p. 345 . 